\section{Wann ist ein Mann ein Mann?}

\section{Die Zugehörigkeit zum männlichen Geschlecht ist offensichtlich nicht nur eine rein biologische Zuordnung, sondern vielmehr ein Status, der ständig verteidigt werden muss. Zu dieser Schlussfolgerung kommen jedenfalls US-amerikanische Psychologen.}

n einer Studie sollten männliche Probanden entweder Zöpfe oder Seile flechten. Das Flechten von Zöpfen wurde von den Probanden als unmännlich empfunden, das Flechten von Seilen hingegen als wertfreie handwerkliche Tätigkeit. Anschließend durften die Versuchspersonen wählen, ob sie ein Boxtraining am Sandsack absolvieren wollten oder lieber ein Puzzle lösen. Die Probanden, die vorher „unmännlich“ Zöpfe geflochten hatüberwiegend den Sandsack. Dort schlugen sie signifikant härter zu als die Seilflechter. Die Aggressivität sollte den Makel, den ihre Männlichkeit durch die Flechterei erlitten hatte, wieder wettmachen. ten, wählten
In einem zweiten Versuch wurde männlichen und weiblichen Pro-

banden ein fiktiver Polizeibericht vorgelegt: In einer Bar war es zu einer Schlägerei gekommen. In einer Version hatte ein Mann einen anderen Mann zusammengeschlagen, weil dieser seine Männlichkeit bezweifelt hatte. In der zweiten Version waren sich zwei Frauen in di e Haare geraten. Die Versuchspersonen w u r d e n nun gebeten, die Persönlichkeit der Aggressorin oder des Aggressors zu bewerten. Die schlägernde Frau wurde von männlichen Versuchspersonen als „unreif “ abgekanzelt, während dem schlägernden Mann zugestanden wurde, er sei in seiner Männlichkeit beleidigt worden und habe diese verteidigen müssen. Frauen beschieden hingegen sowohl der Schlägerin als auch dem Schläger eine unreife Persönlichkeit.

Kommentar: Männlichkeit ist offensichtlich ein Attribut, das nicht nur durch die rein biologische Geschlechtszugehörigkeit definiert ist. Passt Mann nicht auf, ist die Männlichkeit sehr schnell beschädigt, etwa durch soziale Interaktionen. Depressionen, Ängste, niedriges Selbstbewusstsein und Gewalttätigkeit sind die Folgen der ramponierten Männlichkeit. Die Weiblichkeit gerät eher durch körperliche Veränderungen in Gefahr, beispielsweise in den Wechseljahren, meinen die Autorinnen (!) dieser Studie.

Prof. Kurt Malberg

Bosson JK \& Vandello JA. Precarious Manhood and Its Links to Action and Aggression. Curr Dir Psych Sci 2011; 20: 82-6

\section{Häufiger Gendefekt schwächt Spermien}

In Deutschland bleibt jede sechste bis siebte Ehe kinderlos. In jedem zweiten Fall ist die Unfruchtbarkeit des Mannes die Ursache. Bei 70\% der Männer lässt sich die Unfruchtbarkeit nicht mit der Zahl und Qualität der Spermien erklären. US-amerikanische Forscher haben jetzt herausgefunden, dass ein Gendefekt die Fruchtbarkeit dieser Männer vermindern könnte.

E in Forscherteam aus den USA hat eine Genvariante des glykosylierten Polypeptids $\beta$-Defensin 126 (DEFB 126) untersucht, die vom Nebenhoden stammt, sich während ihrer Reifung auf die Oberfläche der Spermien anlagert und für eine optimale Wanderung durch die Flüssigkeiten des weiblichen Genitale sorgt. Bei der Untersuchung von 500 verheirateten chinesischen Paaren fanden die Forscher in der Sequenzvariante DEFB 126 zwei Nukleotiddeletionen, die eine anormale mRNA produzierten. In der chinesischen Population lag die Allelfrequenz bei 0,45, bei Europäern wurde 0,47 gemessen. Ihre Spermien unterschieden sich nicht von denen Gesunder. Sie hatten aber die Eigenschaft, eine dem Gebärmutterschleim ähnliche Testsubstanz schlechter $z u$ durchdringen.
Kommentar: Der neu gefundene Gendefekt ist weit verbreitet. Etwa ein Fünftel der untersuchten Männer aus Asien, Europa und Afrika trägt ihn auf beiden Chromosomen und besitzt dadurch kein normales $\beta$-Defensin. Ihre Spermien haben es schwerer, zu den Eizellen vorzudringen, die betroffenen Männer sind subfertil. Die Entdeckung der Genvariante kann möglicherweise zu einer besseren Behandlung unfruchtbarer Paare beitragen.

Prof. Kurt Malberg

Tollner TL et al. A common mutation in the defensin DEFB126 causes impaired sperm function and subfertility. Sci Transl Med 2011; 3, 92ra65 